This is a peer-reviewed, accepted author manuscript of the following research article: Cameron, J. M., Butler, H., Anderson, D. J., Christie, L., Confield, L., Spalding, K. E., Finlayson, D., Murray, S., Panni, Z., Rinaldi, C., Sala, A., Theakstone, A. G., \& Baker, M. J. (2020). Exploring pre-analytical factors for the optimisation of serum diagnostics: progressing the clinical utility of ATR-FTIR spectroscopy. Vibrational Spectroscopy, 109, [103092]. https://doi.org/10.1016/i.vibspec.2020.103092

\title{
Exploring pre-analytical factors for the optimisation of serum diagnostics: progressing the clinical utility of ATR-FTIR spectroscopy
}

\section{Abstract}

Data quality and reproducibility are vital for robust, reliable and consistent diagnostic techniques within clinical environments. Most variance within clinical laboratories occur within the pre-analytical phase, prior to obtaining and analysing data. Herein, we investigate pre-analytical considerations for the spectroscopic analysis of blood serum for the purpose of clinical diagnostics.

Variables within sample collection, storage and preparation are explored in order to evaluate their effects on the spectral outcome, including; differences between sample collection tubes, centrifugal speed and time, short and long-term storage conditions, individual analyst technique, sample volumes, environment of sample batches, as well as various drying techniques. Exploratory data analysis using principal component analysis was implemented to unearth spectral variance not immediately observable.

Minor spectral variations were observed within each experiment; however, these were not considered significant differences as a result of these varying experimental factors. Variation between different operator techniques when preparing samples was observed, yet can be resolved with appropriate standard operating procedures, regardless of other factors such as sample volume and storage conditions. The findings within this report suggest that experimental variations within a laboratory, or between different laboratories, does not significantly affect the spectral outcome, and with good laboratory practice and careful sample handling results should be consistent and reliable. 


\section{Introduction}

The quest for detecting disease states using biological fluids, or "biofluids", via photonic approaches is a fast-emerging field [1]. Vibrational spectroscopic techniques, such as Fourier transform infrared spectroscopy (FTIR), have been thoroughly investigated in the diagnostics discipline, with many studies presenting statistics that show real promise for their application in a clinical environment [2]. It has been well documented that FTIR spectroscopy can characterise and quantify the levels of specific biomolecules present within biological samples, generating a biochemical fingerprint as the resulting spectrum [3]. The technique has demonstrated high accuracy, being able to detect subtle changes in a patient's blood composition when combined with computational analysis and machine learning algorithms [4]. Most of the biofluid spectroscopy research has focused on blood serum and plasma, due to the prevalence of these types of samples within current biobank stocks. Thus, large retrospective patient cohorts are now feasible with the use of blood as the major diagnostic medium [5]. The ability to diagnose disease rapidly with high sensitivity and specificity would improve the quality of life and prognosis for patients. The collection of blood samples is simple and relatively non-invasive; thus, serum tests are already commonly employed in clinical diagnostics. Therefore, the implementation of a spectroscopic blood test into clinics would not significantly disrupt current diagnostic pathways. One advantage using blood serum is the ability to monitor disease and/or treatment progression, which could ultimately reduce mortality and morbidity.

Many proof-of-concept studies have emphasised the ability of FTIR to differentiate various diseases and malignancies from healthy controls through the spectral 'biosignatures' generated from blood-derived biofluids. The serum and plasma profiles of patients suffering from breast, ovarian, bladder, oesophageal and brain cancers, as well as non-malignant diseases such as Alzheimer's, have been recently examined [6-14]. Despite an abundance of exciting results, researchers have found it extremely difficult to transition from benchtop to clinic through 
issues such as lack of consistency and standardisation of protocols in these studies [15]. Multi-centre studies are now being conducted within the clinical spectroscopy field, hence there is a crucial need to standardise and validate the modalities of sample collection, storage and spectral acquisition $[16,17]$.

Before reaching the spectrometer, there are various critical points that could affect the sample and resultant spectroscopic analysis if they have not been performed efficiently. Methods of blood collection and separation, storage conditions, freeze-thaw cycles, sample preparation, inter-operator errors are just a few of the pre-analytical considerations that could potentially affect the quality and reproducibility of IR spectra. For clinical testing in general, it is believed that most errors occur in the pre-analytical phase. A recent study reported $61.9 \%$ of variances are introduced prior to analysis, compared to $15 \%$ intra-analytical and $23.1 \%$ post-analytical [18]. In order to realise the full potential of FTIR spectroscopy for regular use in healthcare facilities, these questions need to be addressed and understood prior to translation [17].

Sample preparation also has a major influence in the reproducibility of FTIR spectra. Depending on the sampling mode chosen for FTIR analysis - namely transmission, transflection or attenuated total reflection (ATR) - the preparation requirements will vary considerably and should be tailored to the needs of the equipment. For example, there are many IR transparent windows that are suitable for transmission measurements, as well as various internal reflection elements (IREs) to choose from for ATR-FTIR spectroscopy.

In terms of sample collection and storage, there are several points at which variance can be introduced. The majority of hospitals and clinics use venepuncture procedures to collect blood samples, which draws blood from one of the superficial veins in a patient's arm [19]. The generic process is similar worldwide, but there are slight differences depending on the site of collection. Blood will normally be drawn using vacuum extraction systems; however, the type of collection tube can vary between manufacturers. Health services have the choice between tubes that contain different separating gels for serum, and a range of clotting activators for 
plasma. Likewise, some healthcare sites may opt to use the syringe-needle technique, which can be useful for elderly patients with fragile veins [20]. After collection, whole blood is spun in a centrifuge to separate the serum/plasma components. Currently the centrifugation conditions are not consistent, with recommendations ranging from 10-20 minutes in time and $1500-3000 \mathrm{~g}$ in speed $[21,22]$. Haemolysis of red blood cells is a common issue that can occur after centrifugation, that is known to interfere with many tests in clinical chemistry [23,24]. The temperature and duration of time that blood samples can be stored is another matter of debate. In general, it is important to store biospecimens in mechanical freezers at extremely low temperatures to prevent degradation and the destruction of important biomolecules, such as proteins and nucleic acids [25]. Many biochemistry laboratories in the UK utilise $-80^{\circ} \mathrm{C}$ freezers for long term storage of blood-based products, but this is not currently standardised globally $[26,27]$. Some studies have described storing serum at $-20^{\circ} \mathrm{C}$, which is thought to be detrimental to their integrity [28]. It has also been suggested that storage time can have a damaging effect on these types of biospecimens, which could potentially introduce unwanted variance in retrospective spectroscopic research [29]. Repetitive freezing and thawing of plasma and serum has also been examined recently; Lee et al. reported distinct changes in protein concentrations with increased freeze-thaw cycles, and suggested these should be minimised in order to avoid discrepancies in future clinical testing [30].

When analysing biofluids, it is common practice to dry the liquid drops on to a substrate to overcome the spectral interference of water. As water is a strong absorber of IR light, it exhibits a large absorbance band $\left(\sim 1640 \mathrm{~cm}^{-1}\right)$ which can obscure biological information in the fingerprint region of the IR spectrum $\left(1800-1000 \mathrm{~cm}^{-1}\right)$ [31]. One downfall of this method is that biofluid deposits tend to dry heterogeneously which results in an array of complex patterns, such as the well-known coffee ring effect [32]. Capillary flow forces biomolecules to move to the periphery of the drop as the liquid evaporates, leaving a ring-like shape behind [33]. Bonnier et al. showed this issue can cause spectral differences across the dried droplet; 
observing peak shifts and alterations in band intensity ratios between the drop's edge and its centre [31]. Gelation and cracking patterns are also commonly observed when dehydrating blood serum onto IR substrates [34]. Hughes et al. measured 20 single-point transmission spectra over a single blood serum deposit; cracks throughout the droplet were shown to cause scattering effects, as well as varied absorbances in the Amide I/II region (1700-1500 $\left.\mathrm{cm}^{-1}\right)$ [35]. Recently there has been some preliminary work performed to understand and overcome such pre-analytical variances. Lovergne et al. carried out a dilution study with blood serum to try and control the complex drying patterns. Using transmission mode and FTIR imaging, it was found that a 3 -fold dilution was the optimum concentration to minimise the cracking patterns that was observed in whole serum. However, coffee rings were still apparent in the diluted samples, with the outer edges of the drops exhibiting higher absorbance values [36]. Moreover, the addition of a dilution step prior to spectral analysis would likely be unfavourable for clinical translation. A later study looked into several pre-analytical factors, such as the biofluid volume and dilution, sample collection modality and the impact of freeze-thaw cycles on spectral reproducibility [15]. The authors highlighted that the development of an automated device for data acquisition could be more attractive to clinical laboratories. Furthermore, it was concluded that the optimal pre-analytical procedure will be determined by the type of biofluid to be analysed and the sampling mode that is available. Recently, additional pre-analytical factors were examined, such as storage tube capacity and inter-aliquot reproducibility of serum and plasma samples, which suggested that neither the volume of sample stored nor the storage tube capacity has an impact on spectral reproducibility [37]. Standardisation of sample collection parameters is an important requirement in the regulatory approach for all diagnostics methods and the authors point the readers to the following resource to understand the challenges associated with this [27].

In this study, we build on these previous findings with three main objectives: to determine the effect of (i) sample collection (collection tube type, centrifugation parameters); (ii) sample 
storage (short- and long-term); and (iii) sample preparation (intra- and inter-operator variability, volume of serum and impact of drying conditions/environment/humidity) on spectra data. The overall aim is to of decipher the optimum sample collection, storage and preparation parameters for the analysis of blood serum with ATR-FTIR spectroscopy, to enable successful clinical translation of the technology.

\section{Materials and Methods}

Unless otherwise stated in the experimental design (Table 1), the following procedure was implemented for each of the analyses. The full experimental design for each analysis is outlined in the Supplementary Information (SI). A general overview of the study is described in the schematic in Figure 1.

\section{Blood serum collection}

Participant enrolment and sample collection from healthy volunteers was carried out with NHS ethical approval (NHS North-West - Preston Regional Ethical Committee (REC); REC Reference: 18/NW/0288, Protocol Number: UEC 18/21; date awarded 15/08/18) and with NHS Research and Development and Board Approval (NHS Greater Glasgow \& Clyde Research and Development, Ref: GN18ON143, Date awarded 15/08/18) and in accordance with the principles set out in the declaration of Helsinki. Blood was sampled using the standard venepuncture technique. The Greiner Bio-One Gold Top VACUETTE® TUBE 5 mL CAT Serum Separator Clot Activator blood collection system (Greiner Bio One, Austria) was used for blood sample collection. $2 \mathrm{~mL}$ of whole blood was collected from each participant. Each blood collection tube was gently inverted following sampling to encourage even mixing whilst avoiding haemolysis. Samples were left to clot at room temperature for 30 minutes prior to centrifugation for component separation, which was performed at $4100 \mathrm{~g}$ for 5 minutes, according to local protocols. Commercially available mixed human pooled serum was 
purchased from TCS Biosciences, UK. All serum samples were frozen to $-80{ }^{\circ} \mathrm{C}$ for storage prior to analysis.

\section{Sample preparation}

The serum samples were removed from storage and allowed to thaw for 15-20 minutes. $3 \mu \mathrm{L}$ was pipetted onto each of the three sample wells of a disposable optical slide (ClinSpec Diagnostics Ltd, UK), leaving the first well clean for background collection [12]. The serum drops were spread evenly across the sample wells using the pipette tip, ensuring the full well was covered to create a thin serum film. Prepared slides were stacked in $3 \mathrm{D}$ printed polylactic acid slide holders, which were designed to enable batch drying. The stacked slides were then stored in a drying unit incubator (Thermo Fisher ${ }^{\mathrm{TM}}$ Heratherm $^{\mathrm{TM}}$, GE) at $35^{\circ} \mathrm{C}$ for an hour to control the drying dynamics of the serum drop [34].

\section{Spectral collection}

IR spectra were collected in the range of $4000-450 \mathrm{~cm}^{-1}$ at a resolution of $4 \mathrm{~cm}^{-1}$ with 16 co-added scans. A background spectrum was performed which was automatically subtracted from the acquired sample spectra. Three spectra were acquired for each biological replicate and three technical replicates were performed, resulting in nine spectra for all samples. The environmental conditions from all investigations ranged between $18-23{ }^{\circ} \mathrm{C}$ and humidity between 15-35\%. All spectra were recorded on either an Agilent Cary 600 FTIR spectrometer (Agilent Technologies, USA) or PerkinElmer Spectrum 2 FTIR spectrometers (PerkinElmer, UK), fitted with a Specac Quest ATR (Specac Ltd, UK), with either a diamond IRE (DIRE) top plate, or a silicon IRE (SIRE) (ClinSpec Diagnostics Ltd, UK) using a specular reflectance puck.

\section{Spectral data analysis}

Data was pre-processed and analysed using Matlab with a graphical user interface which was developed in-house by the Spectral Analytics Laboratory, University of Strathclyde. The 
processed IR spectra were cut to the biologically relevant region for each experiment, with the typical fingerprint region being $1800-1000 \mathrm{~cm}^{-1}$. This was then followed by rubberband baseline correction and vector normalisation for all processed spectra. Principal component analysis (PCA) was conducted and PC loadings were employed to identify the origin of spectral variance.

At the sample collection and storage, as well as the sample preparation stages of this experiment, consideration was given to several variables that could affect the reliability and consistency of the results. Figure 1 describes the experimental plan, and Table 1 outlines the processes where one variable was altered (shaded in grey) whilst all other experimental conditions were kept consistent. Full details are included within the SI. 


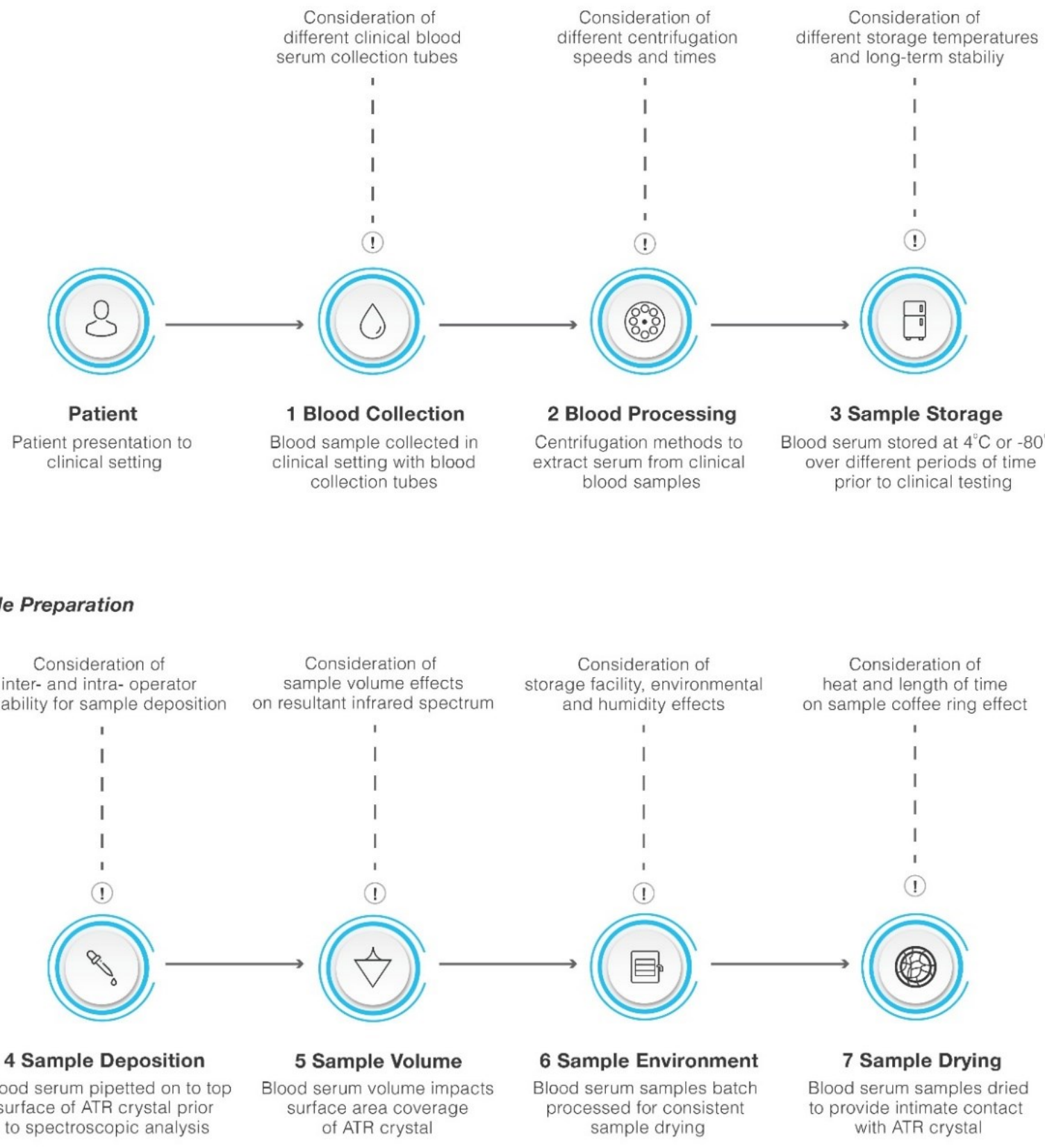

Figure 1: Experimental protocol flowchart outlining each consideration at every step of the sample collection,

storage and preparation stages. 
Table 1 - Experimental conditions for each step of the analytical process labelled 1-7. The variable under examination is highlighted in grey. Full experimental details

\begin{tabular}{|c|c|c|c|c|c|c|c|c|c|c|c|}
\hline \multirow{2}{*}{$\begin{array}{l}\text { Experimental } \\
\text { process }\end{array}$} & \multirow{2}{*}{$\begin{array}{c}\text { Sample type } \\
\text { (pooled or patient) }\end{array}$} & \multirow{2}{*}{$\begin{array}{l}\text { Blood collection } \\
\text { tube }\end{array}$} & \multicolumn{2}{|c|}{ Centrifuge } & \multicolumn{2}{|c|}{ Storage } & \multirow{2}{*}{$\begin{array}{l}\text { Number } \\
\text { of } \\
\text { operators }\end{array}$} & \multirow{2}{*}{$\begin{array}{l}\text { Sample } \\
\text { volume } \\
(\mu \mathrm{L})\end{array}$} & \multirow{2}{*}{$\begin{array}{c}\text { Sample } \\
\text { environment } \\
\text { (after drying } \\
\text { period) }\end{array}$} & \multirow{2}{*}{$\begin{array}{l}\text { Sample } \\
\text { drying } \\
\text { technique }\end{array}$} & \multirow{2}{*}{$\begin{array}{c}\text { IRE } \\
\text { (diamond or } \\
\text { silicon) }\end{array}$} \\
\hline & & & $\begin{array}{l}\text { Time } \\
(\mathrm{min})\end{array}$ & $\begin{array}{l}\text { Speed } \\
(\mathrm{g})\end{array}$ & $\begin{array}{l}\text { Short- } \\
\text { term } \\
\text { (s.t) }\end{array}$ & $\begin{array}{l}\text { Long-term } \\
\text { (1.t) }\end{array}$ & & & & & \\
\hline 1 & Patient & $\begin{array}{l}\text { BD Vacutainer SST } \\
\text { II tubes, Vacuette } \\
\text { serum tubes with } \\
\text { gel clot activator } \\
\text { and Monovette } \\
\text { serum gel Z } \\
\text { separation tubes }\end{array}$ & 5 & 4100 & $-80^{\circ} \mathrm{C}$ & $-80^{\circ} \mathrm{C}$ & 1 & 3 & Benchtop & Incubator & Silicon \\
\hline 2 & Patient & $\begin{array}{l}\text { Vacuette serum } \\
\text { tubes with gel clot } \\
\text { activator }\end{array}$ & $5-15$ & $\begin{array}{l}1635- \\
4186\end{array}$ & $-80^{\circ} \mathrm{C}$ & $-80^{\circ} \mathrm{C}$ & 1 & 3 & Benchtop & Incubator & Silicon \\
\hline 3 & $\begin{array}{l}\text { Patient (s.t) } \\
\text { Pooled (1.t) }\end{array}$ & $\begin{array}{l}\text { Vacuette serum } \\
\text { tubes with gel clot } \\
\text { activator (s.t) }\end{array}$ & 5 & 4100 & $4{ }^{\circ} \mathrm{C}$ & $-80^{\circ} \mathrm{C}$ & 1 & 3 & Benchtop & Incubator & $\begin{array}{c}\text { Silicon (s.t) } \\
\text { Diamond (1.t) }\end{array}$ \\
\hline 4 & Pooled & NA & 5 & 4100 & $-80^{\circ} \mathrm{C}$ & $-80^{\circ} \mathrm{C}$ & $1-7$ & 3 & Benchtop & Incubator & Silicon \\
\hline 5 & Pooled & NA & 5 & 4100 & $-80^{\circ} \mathrm{C}$ & $-80^{\circ} \mathrm{C}$ & 1 & $1-10$ & Benchtop & Incubator & Silicon \\
\hline 6 & Pooled & NA & 5 & 4100 & $-80^{\circ} \mathrm{C}$ & $-80^{\circ} \mathrm{C}$ & 1 & 3 & $\begin{array}{l}\text { Incubator, } \\
\text { desiccator and } \\
\text { benchtop }\end{array}$ & Incubator & Silicon \\
\hline 7 & Pooled & NA & 5 & 4100 & $-80^{\circ} \mathrm{C}$ & $-80^{\circ} \mathrm{C}$ & 1 & 3 & Benchtop & $\begin{array}{l}\text { Air dried, } \\
\text { fan assisted } \\
\text { and heating }\end{array}$ & $\begin{array}{l}\text { Silicon and } \\
\text { Diamond }\end{array}$ \\
\hline
\end{tabular}

available in Supplementary Information. 


\section{Results and Discussion}

\section{Blood collection}

Multiple repeats of a single donor patient, whose blood was collected using three types of clinical serum collection tubes, were used in order to observe any tube-specific variation. Firstly, a PCA-based quality test at a 95\% confidence interval was carried out on the spectral data. From a total of 135 spectra, three spectra were projected outside the confidence interval and were then removed from further analysis (Figure S1 in SI).

The PCA scores plot between PC1 and PC2 (Figure S2a) describes the general variation in the dataset. PC1 accounts for $61.3 \%$ of the variance, which is described by the loadings plot in Figure S2b, in the SI. The PC1 variance mostly relates to the $\mathrm{CO}$ and $\mathrm{CN}$ stretching, and $\mathrm{NH}$ bending vibrations arising from the proteinaceous components in the Amide I and II bands, at around $\sim 1650 \mathrm{~cm}^{-1}$ and $1550 \mathrm{~cm}^{-1}$, respectively. The spectral data points for each tube type are widely spread across both PC1 and PC2, and there are no noticeable clusters within the three tube classes. Yet when the higher PCs are considered, such as PC4 against PC5, the data points begin to group together (Figure 2a), which demonstrates there may be minor spectral differences between the tube types. The loadings for PC4 and PC5 (Figure 2b and 2c) again highlight discrepancies in the Amide I and II bands, as well as noteworthy variations in the 1100-100 $\mathrm{cm}^{-1}$ region, which can be tentatively assigned to carbohydrate (CO stretch), glycogen ( $\mathrm{CO}, \mathrm{CC}$ stretch, $\mathrm{COH}$ deformation) and phosphate $\left(\mathrm{PO}^{2-}\right.$ stretch) vibrations [38]. However, PC4 and PC5 only account for $2.8 \%$ and $1.3 \%$ of the overall variance within the data. 

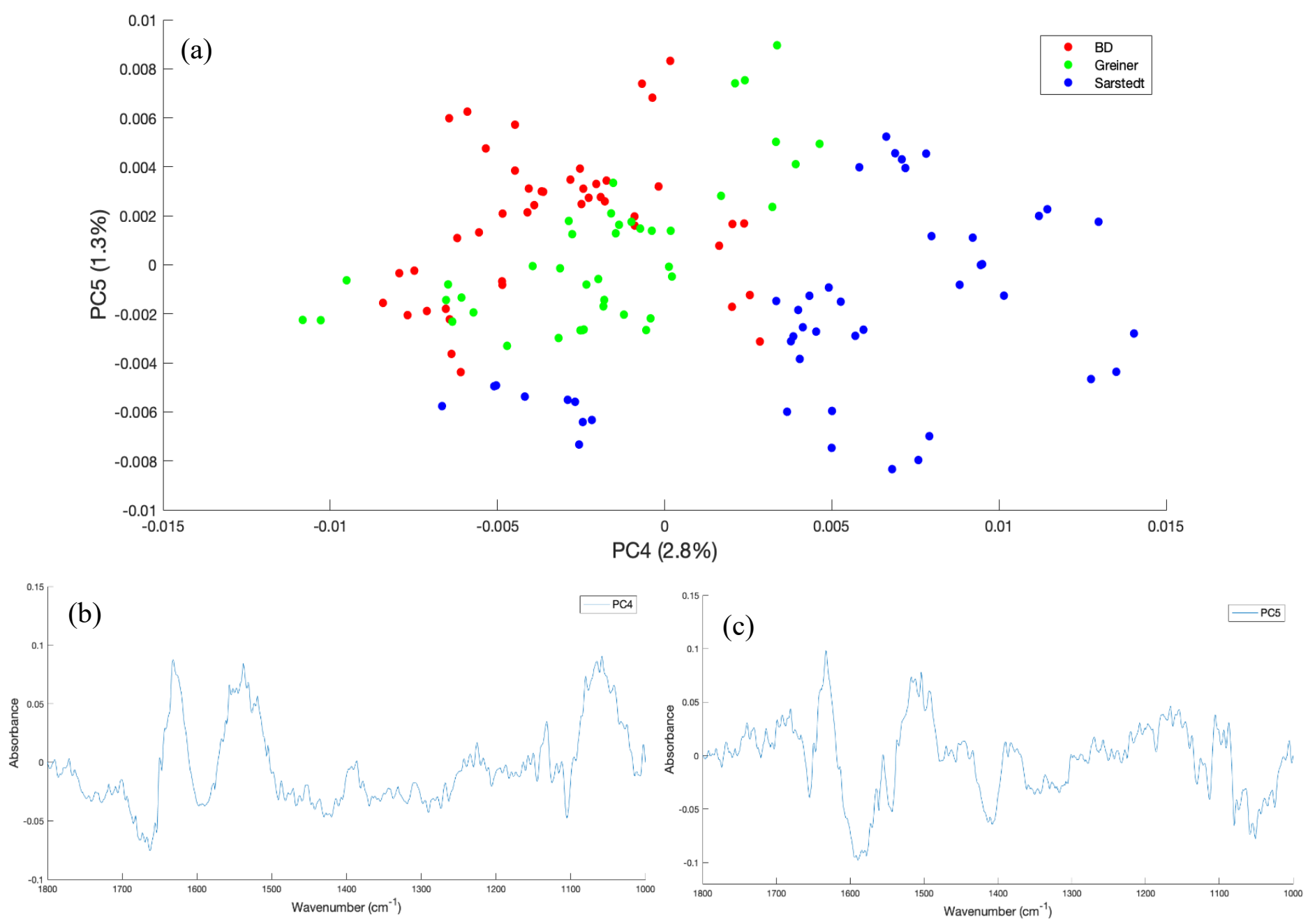

Figure 2: a) PCA scores plot of PC4 and PC5 displaying the variance between the three brands of collection tubes; BD (red), Greiner (green) and Sarstedt (blue), with the corresponding b) PC4 and c) PC5 loadings.

\section{Blood processing}

Serum was successfully obtained from all centrifugation conditions, appearing partially transparent and pale-yellow in colour. PCA showed that there were no real variances among the different centrifugal speeds (rpm) (Figure 3a). The first PC amounts to 29.2\%, however there is no distinct clustering of the different parameters. These results are confirmed by the mean spectral plot with standard deviations included (Figure 3b), which highlights the regions with the greatest variation in the spectra. Ultimately, there are no substantial differences 
between samples, thus if serum is successfully extracted then spectral response is relatively uniform. The minor differences reflected in the PC2 scores plot (accounting for only $18.6 \%$ of the cumulative variance) can be clearly identified as differences in the substrate spectral response (please see Figure S10) and not of biological origin.

(a)
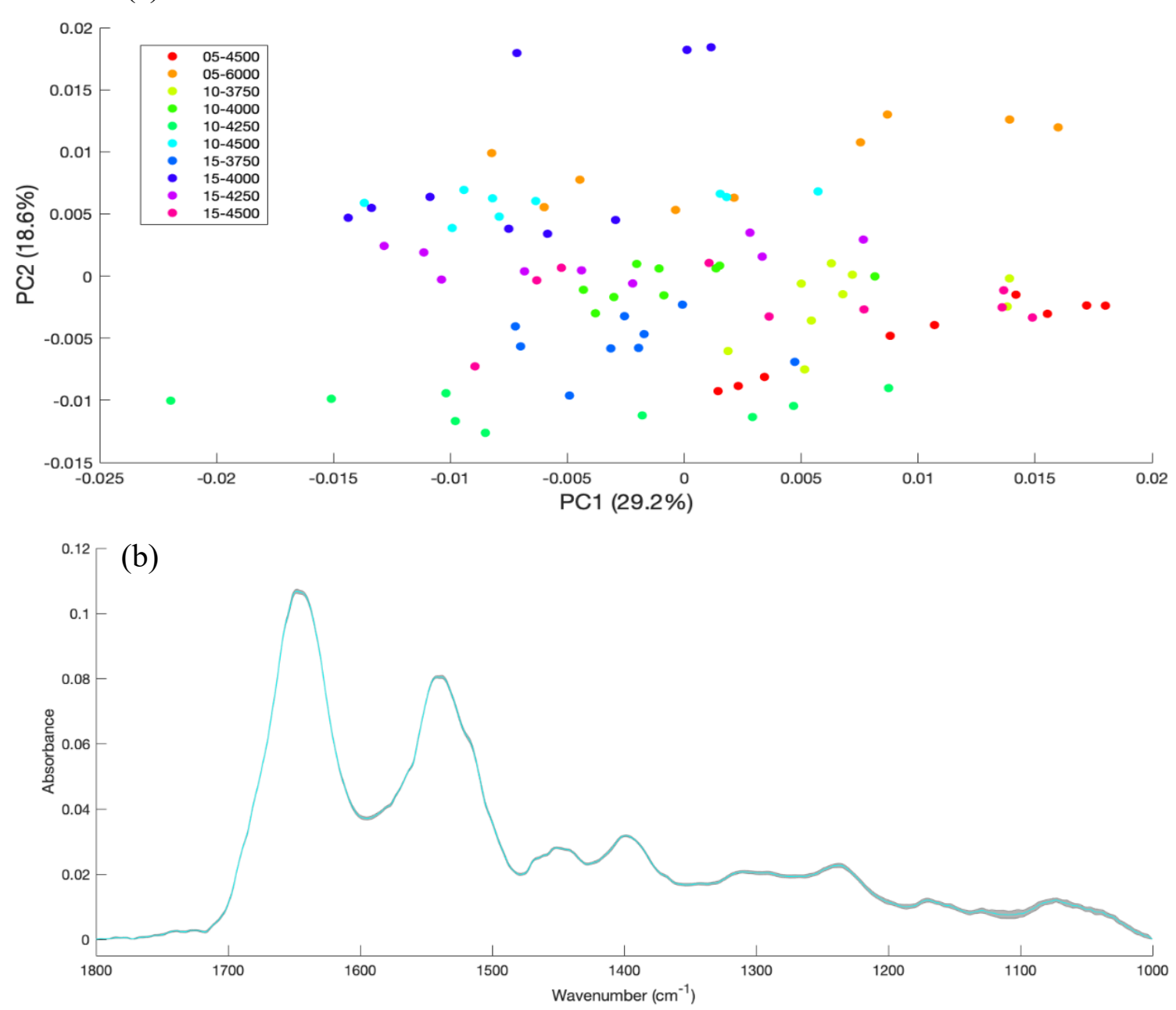

Figure 3: a) PCA scores plot PC1 and PC2 showing minimal variance in the different times and speeds of centrifugation samples and b) mean IR spectra (blue) with the standard deviation highlighted in grey.

\section{Sample storage}

3.1 Short-term storage

The first part of this section looked at the potential flaws of storing freshly extracted serum at

$4{ }^{\circ} \mathrm{C}$. Whilst not recommended, it is not unusual for researchers in clinical laboratories to hold 
serum samples in the refrigerator many hours, up to a few days, before transferring to $\mathrm{a}-80{ }^{\circ} \mathrm{C}$ freezer, often due to logistical reasons. For example, the freezer could be located at a different department or facility. Since many laboratories make blood samples available for general use as a waste product after they have completed their analysis, this could be a confounding factor in many research studies when serum specimens are obtained from certain biobanks.

When the spectra gathered from one individual patient is examined, the variance between storage states is quite distinct, which corresponds with a previous study [37]. Within the SI, Figure S3a illustrates the separation between frozen and refrigerated for patient 1 who displayed the most significant clustering across all patients within this study. The classes split across PC2, the loadings show that the highest variance exists in the Amide I and II region around $1700-1500 \mathrm{~cm}^{-1}$ (Figure S3b). This could be due to an alteration in global protein concentration and/or changes in protein conformation, implying there may be a lack of stability in some biomolecules when exposed to the higher refrigeration temperatures of $4{ }^{\circ} \mathrm{C}$. These findings are consistent with a previous study that used electrophoresis to compare equine serum, stored at $4{ }^{\circ} \mathrm{C}$ and $-20{ }^{\circ} \mathrm{C}$ for 48 hours, where the total protein content was significantly lower in the refrigerated sample than the frozen specimen $(52.3 \pm 3.0 \mathrm{~g} / \mathrm{L}$ c.f. $67.5 \pm 8.7 \mathrm{~g} / \mathrm{L})$ [39]. Yet when we explore multiple patients these variances become less obvious (Figure 4a). From the PCA scores plot in Figure 4b, it becomes discernible that the individual patients begin to cluster together, rather than splitting based on storage facility. This is an interesting concept, in that factors such as immediate storage facility cause distinct discrepancies when looking at one individual's serum signature, but when we introduce additional patients and expand into larger cohorts - which would generally be the case for clinical spectroscopy - the "more important' biological variances become more emphasised. 


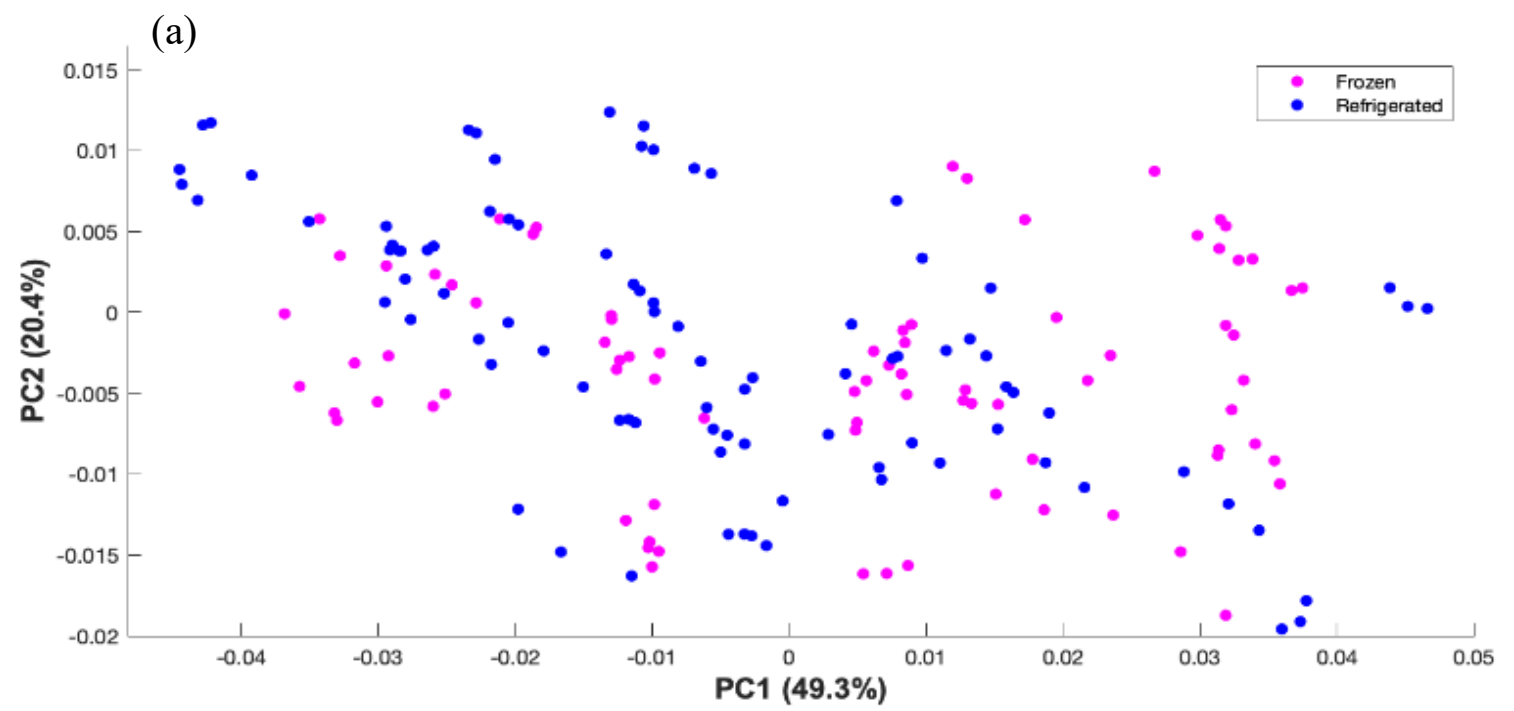

(b)

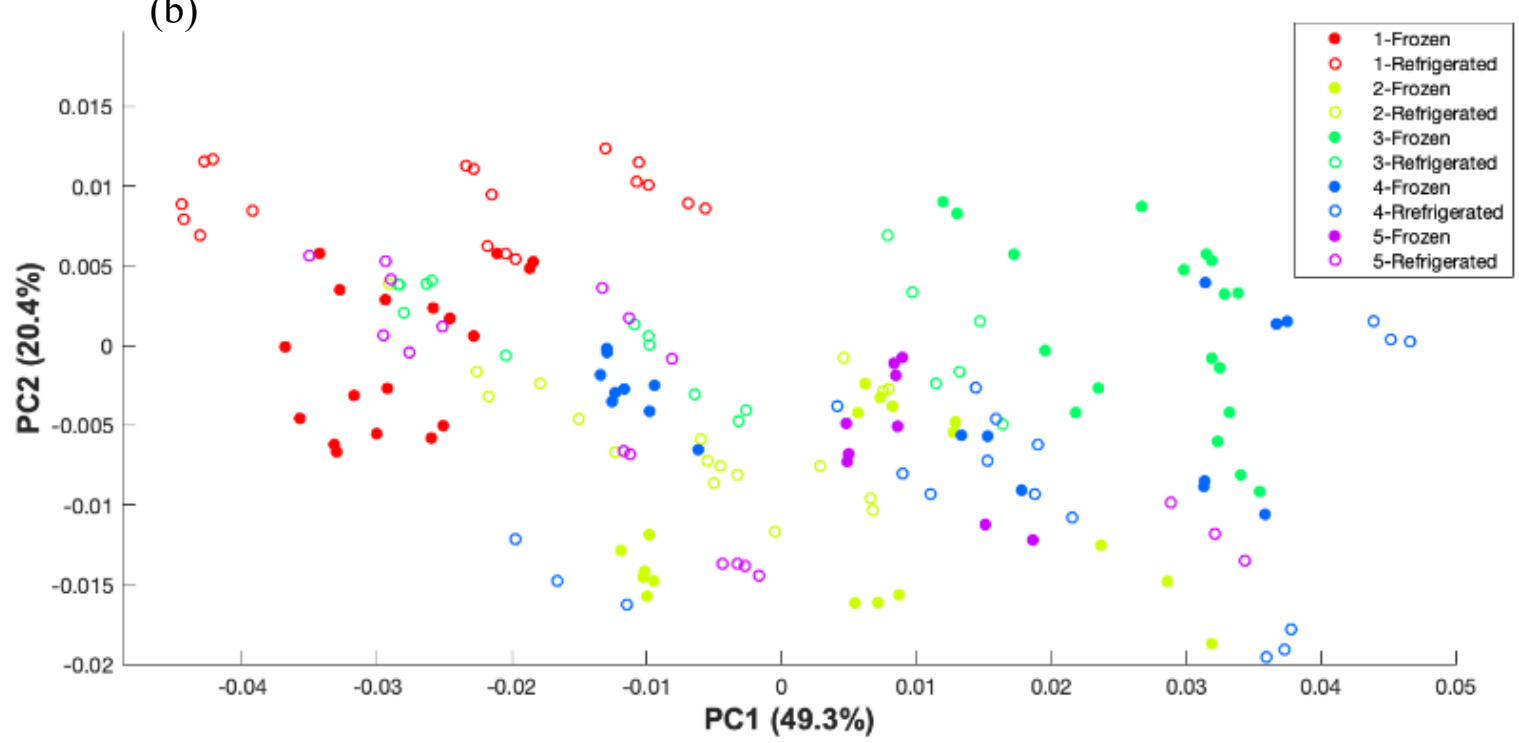

Figure 4: PCA results: a) scores plot of PC1 and PC2 for all 5 patients showing the differences between refrigerated (blue) and frozen (pink), and b) scores plot highlighting the grouping of five individual patients from both refrigerated and frozen short-term storage.

\subsection{Long-term storage}

The second part of this section explores spectra from human pooled serum that was collected over a period of 28 months to determine the impact on long-term storage. Spectra obtained at each of the tested time-points, as shown in the SI (Figure S4) were converted to second 
derivative. The scores plots (Figure 5a) explain $73.7 \%$ of the total variance by the first two PCs, which is $39.0 \%$ and $34.7 \%$, respectively. The corresponding loadings plot (Figure 5b) shows that the spread across the zero line of PC1 is attributed to the Amide I band between $1700-1600 \mathrm{~cm}^{-1}$ due to the stretching vibrations of the $\mathrm{CO}$ and $\mathrm{CN}$ groups; as well as the Amide II region of $1600-1500 \mathrm{~cm}^{-1}$ resulting from the $\mathrm{NH}$ bending, $\mathrm{CN}$ stretching and the $\mathrm{CC}$ stretching modes.

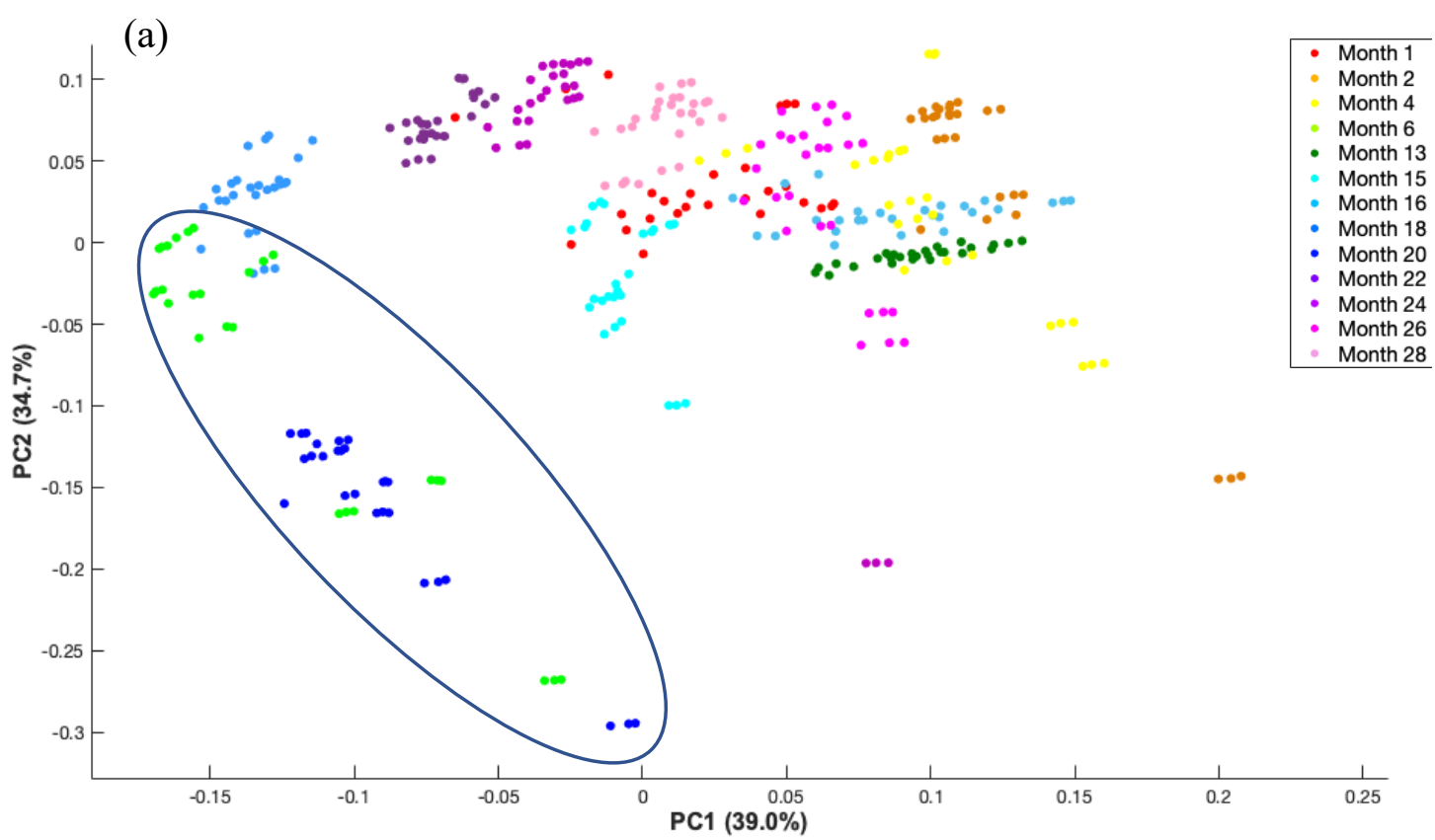

(b)

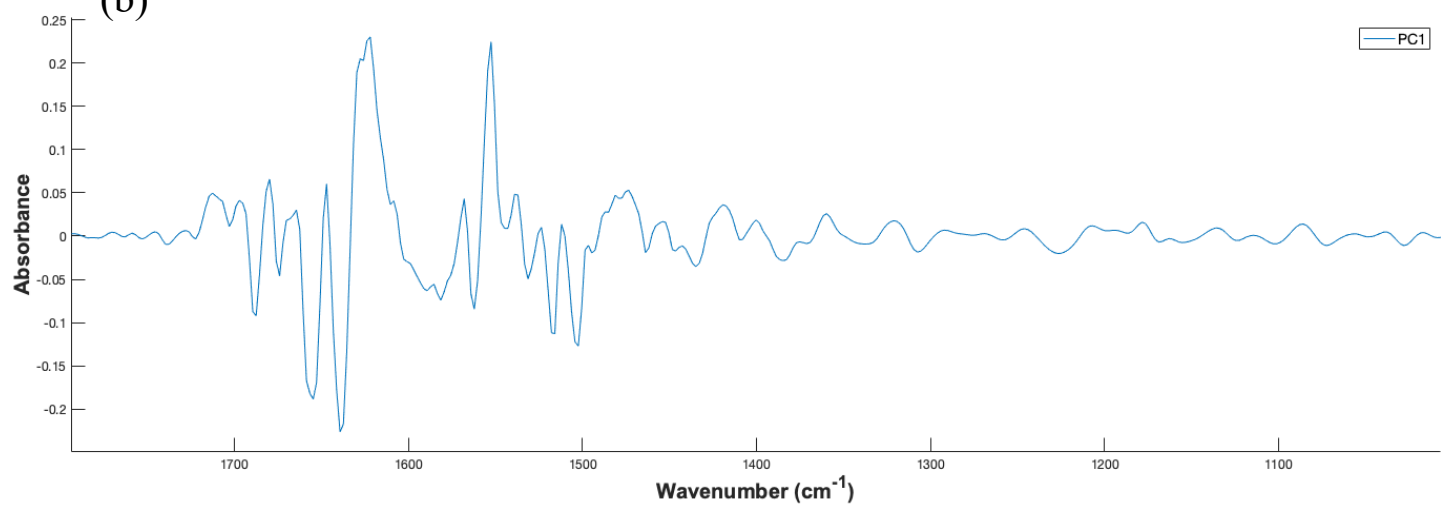

Figure 5: Scores plots for a) PC1 v PC2 with a blue circle highlighting month 6 (green) and month 20 (dark blue) as outliers; and b) the corresponding loadings plot for PC1.

Interestingly, the PCA allows for the identification of specific months, particularly months 6 (green) and 20 (dark blue) as outliers within the data. Through the observation of the loadings plot associated with PC2 an insight as to why this may be the case is not provided (data not 
shown). Further investigation led to the discovery that the humidity during analysis was increased to $59 \%$, opposed to the average of $38 \%$ for this part of the study, potentially resulting in changes to the protein structures within the human pooled serum during analysis, rather than during storage, and could explain these particular outliers. The examination of the long-term storage of human pooled serum sub-samples over a period of 28 months at $-80{ }^{\circ} \mathrm{C}$ suggests that there is no impact on resulting spectral data. Until now, clinical validation such as this was absent from literature and as pointed out by Lovergne et al. [15] was a necessary step to achieve translation.

\section{Sample deposition}

\subsection{Inter- and intra-operator variability}

Seven operators were asked to prepare slides for SIRE analysis using human pooled serum without following a specific SOP in order to observe any spectral variations caused by the sample preparation process. PCA was performed on all data sets, which highlights that minor differences between operators, mainly arises from variances among slide wells (Figure 6). The optimal SD between the three sample wells (lowest value) was operator 5, and the highest value came from the sample slide made by operator 1 (Table 2).

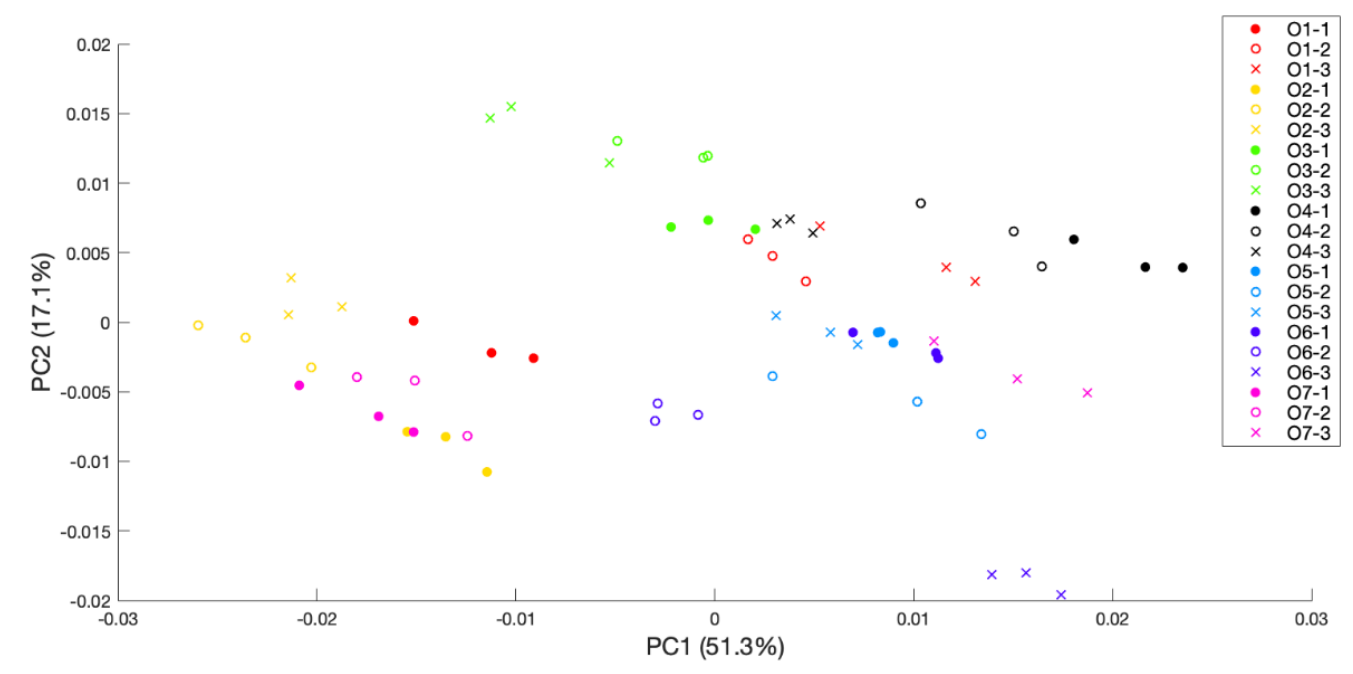

Figure 6: Inter-operator PCA scores plot of PC1 and PC2 showing minor differences between operators. 
Table 2: Average standard deviation values calculated from the mean spectra from the three sample wells for each operator.

\begin{tabular}{|c|l|}
\hline Operator & Average SD \\
\hline $\boldsymbol{1}$ & $5.490 \times 10^{-4}$ \\
\hline $\mathbf{2}$ & $3.715 \times 10^{-4}$ \\
\hline $\mathbf{3}$ & $3.037 \times 10^{-4}$ \\
\hline $\mathbf{4}$ & $4.482 \times 10^{-4}$ \\
\hline $\mathbf{5}$ & $2.582 \times 10^{-4}$ \\
\hline $\boldsymbol{6}$ & $3.785 \times 10^{-4}$ \\
\hline $\boldsymbol{7}$ & $3.951 \times 10^{-4}$ \\
\hline
\end{tabular}

This implies inter-operator variance is likely to occur, and that care must be taken when preparing serum samples for analysis. The operators appeared to use different techniques when spreading the serum drops evenly across the sample wells. The pipetting movement did not seem to impact on the spectral measurement, however the position of immediate deposition on the sampling well ultimately caused varying SD values. Therefore, when using SIREs, it is advised to start from the centre of the IRE, before spreading it to corners and edges if required, covering all empty areas on the surface of the IRE to create a thin serum film. That being said, similar results were observed from the multiple repeats prepared by the individual operator, where some slides had a higher SD than others (Figure S5 and Table S3 in SI). Nevertheless, the SD values are extremely low and are unlikely to be truly significant.

5. Sample volume

Sample volume studies were conducted on silicon substrates with human pooled serum to assess whether increased quantities, therefore increased thicknesses, of biological molecules on the IRE surface influenced spectral responses. Generally, all sample volumes from 1-10 $\mu \mathrm{L}$ 
were observed to produce IR spectra characteristic of human serum, as shown within the SI (Figure S6). All sample volumes reproducibly observed characteristic vibrational modes at wavenumbers that correlated with the presence of protein, carbohydrate, lipid and nucleic acid content within human blood serum [40]. Closer inspection of our preliminary findings tentatively deemed $3 \mu \mathrm{L}$ to be the optimum volume for spectral analysis as it produced the highest signal-to-noise ratio for all sample volumes between 1-10 $\mu \mathrm{L}$ (Figure 7).

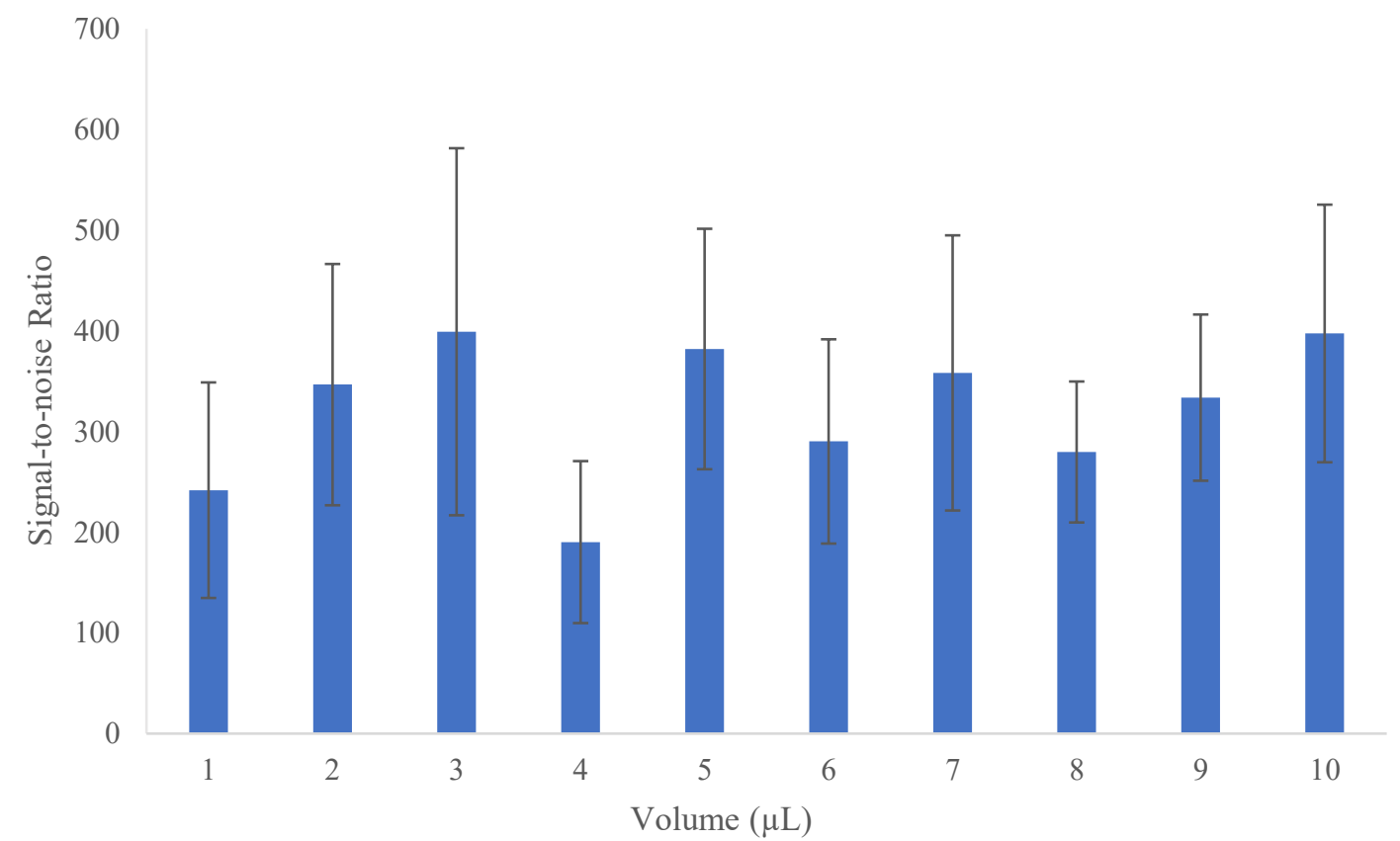

Figure 7: Plot of mean signal-to-noise ratio with associated standard deviations for 1-10 $\mu \mathrm{L}$ sample volumes calculated from intensities of the Amide I peak $\left(1620-1670 \mathrm{~cm}^{-1}\right)$ with respect to the $1850-1900 \mathrm{~cm}^{-1}$ region $(\mathrm{n}=9)$.

However, it should be noted that the standard deviation for $3 \mu \mathrm{L}$ sample volumes was considerably large, albeit consistent with standard deviations of other sample volumes. Furthermore, $3 \mu \mathrm{L}$ was considered the most appropriate sample volume for spectral investigation of human serum, as it was the minimal volume that could reproducibly cover the surface area of the silicon substrate in its entirety. Overall, it is reassuring that different sample volumes and thicknesses do not promote considerable biological spectral variation on silicon 
substrates, which is anticipated for ATR-FTIR given the defined depth of penetration of evanescent waves at $\sim 1-2$ microns in the fingerprint region [40]. Nevertheless, deposition of 3 $\mu \mathrm{L}$ sample volumes were deemed most appropriate for spectral analysis of human serum given the increased practicality and signal-to-noise ratio relative to other tested sample volumes.

\section{Sample environment}

The potential effect of humidity and the environmental conditions that batch processed sample slides are subjected to prior to spectral acquisition was examined. Dehydration of a serum drop results in a reduction of the shoulder between $3600-3300 \mathrm{~cm}^{-1}$ representing $\mathrm{OH}$ vibrational modes due to the loss of water and destruction of hydrogen bonding within the sample [41]. Likewise, the absorbance intensity of the Amide I band tends to increase as biofluids dry directly onto an IRE. Small differences in mean absorbance spectra can be seen due to different drying environments after 5 hours of environmental exposure (Figure 8).

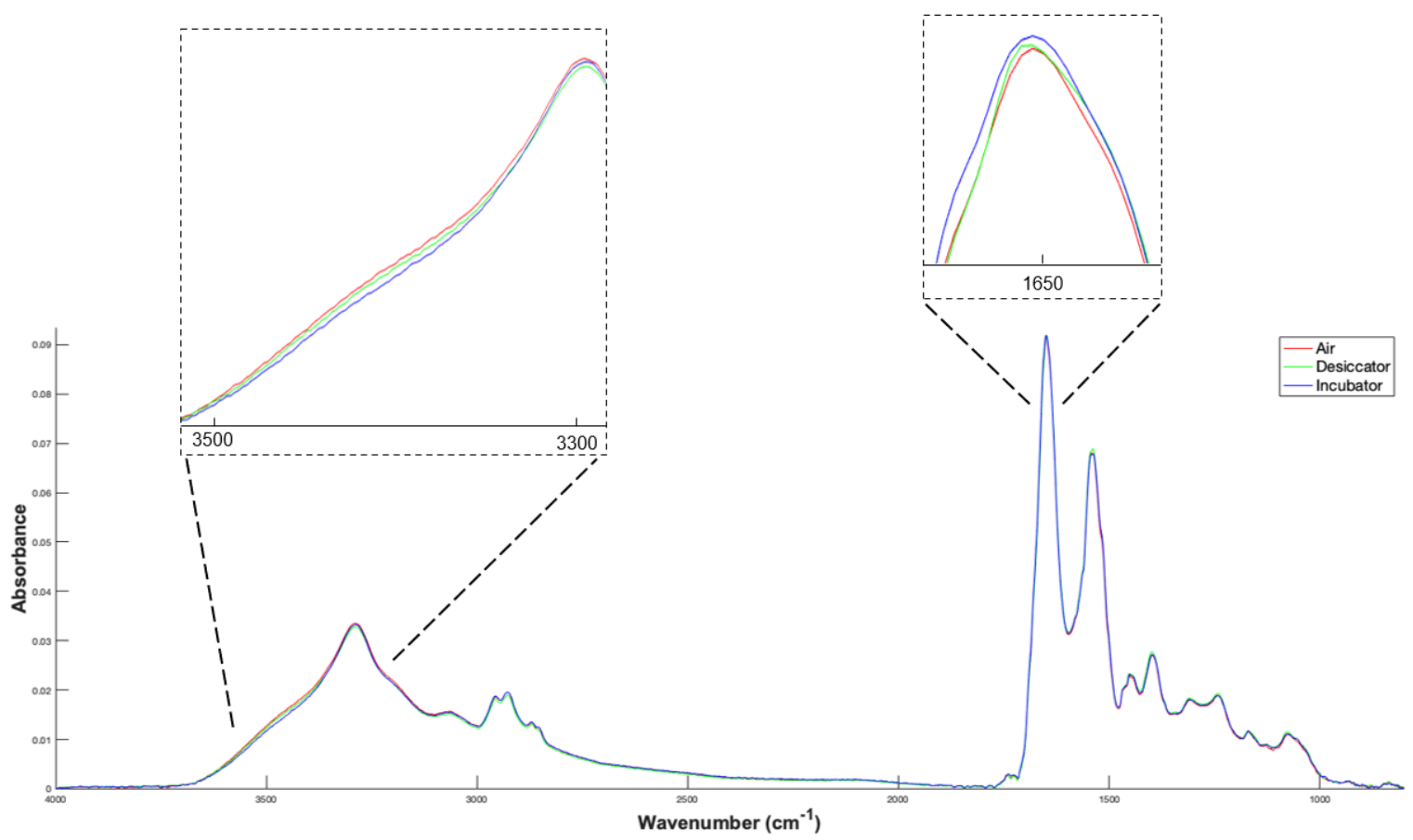

Figure 8: Infrared spectra obtained 5 hours after sample preparation, where samples had been stored in either a heated incubator (blue), desiccator (green) or on the laboratory benchtop in ambient conditions (red). 
The resulting spectra suggests the serum stored in the heated incubator exists in a drier state (blue spectrum) than the samples left on the benchtop and in the desiccator, due to the lower intensity at the $\mathrm{OH}$ stretch $\left(\sim 3400 \mathrm{~cm}^{-1}\right)$ and a slight increase in the Amide I vibration (1650 $\mathrm{cm}^{-1}$ ). This could be explained by the assumption that the prolonged period in a heated environment instigates more intimate sample-IRE contact, thus allowing more efficient penetration of the evanescent wave [42]. However, the difference in mean absorbance is minute at approximately 0.001 , suggesting the variation is inappreciable. Thus, serum sample slides can be left on the benchtop for at least 5 hours before spectral collection, once they have been prepared appropriately.

The slides were then left in their corresponding environments overnight and re-analysed the following day. The spectral datapoints are spread across the PC1 and PC2 scores plot (Figure 9). As expected, the incubated slides appear to cluster together the most (blue scores), mainly due to differences in the Amide bands and $\mathrm{OH}$ stretching vibrations (Figure $\mathrm{S} 7$ in $\mathrm{SI}$ ). Furthermore, as shown in SI Figure S8, there is a considerable drop in intensity after 24 hours

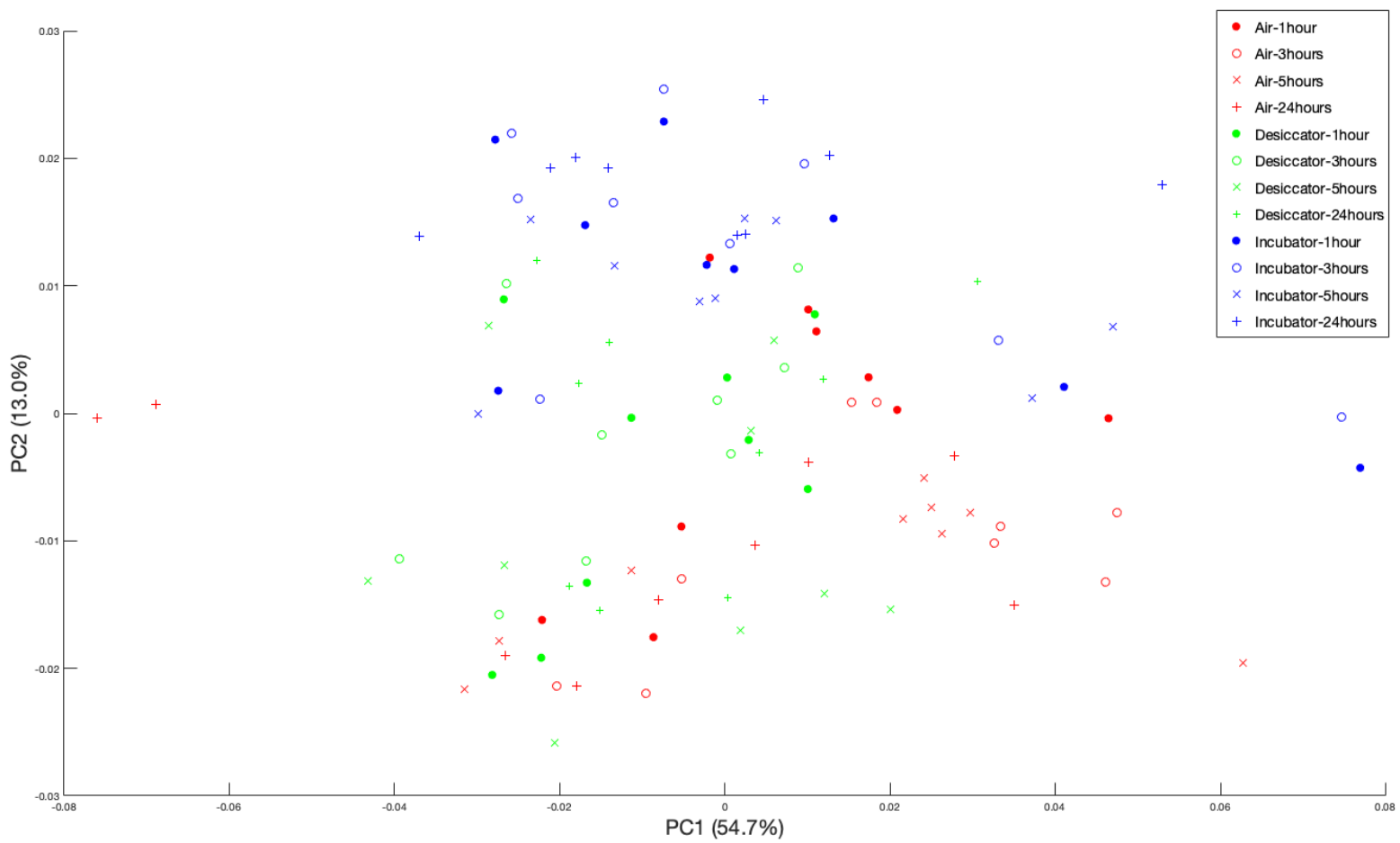


for all three environments (purple spectra), which is likely due to the degradation of various protein structures over time.

Figure 9: PCA scores plot of PC1 against PC2 for the three storage environments analysed after the four timepoints: benchtop (red), desiccator (green) and incubator (blue).

\section{Sample drying}

It is well established that when interrogating serum using ATR-FTIR, the samples must be dried onto the IRE before any analysis can be carried out. With respect to sample preparation, this is an extremely simple practice relative to many other bioanalytical techniques that require many careful steps and expensive reagents. It is preferred to dehydrate the serum drops prior to spectral collection, mainly because water is a strong absorber of infrared light, but also to optimise the sample to crystal contact profile. Water has three vibrational modes which tend to obfuscate concurrent vibrational modes of analyte molecules. Furthermore, in ATR mode water gives rise to anomalous dispersion effects which distort the $\mathrm{OH}$ band and skew quantitative analysis [43]. Thus, here we evaluate different drying environments to determine the optimal protocol for all internal reflection elements.

\subsection{Normal drying on diamond and silicon}

Silicon spectra showed increased absorption of biological bands over the entire mid-infrared wavenumber region with concurrent reductions in water signatures over time (data not shown). Specifically, infrared peaks reached maximum absorption at 12 minutes with increased intensities of the Amide I and II bands in the fingerprint region, and the emergence of Amide $\mathrm{A}$ in the higher wavenumber region, resulting from symmetric and asymmetric $\mathrm{CH}$ stretching vibrations. Spectral features remained unchanged from this time point over an extended period, therefore 12 minutes was deemed sufficient to dry serum on silicon ATR crystals. Spectra obtained on diamond similarly showed increased absorption of infrared peaks over time as serum samples dehydrated in air and were deemed dry for spectroscopic analysis at 18 minutes. 
Figure 10 illustrates the IR spectra collected over a time period of 32 minutes on a diamond IRE, displaying the specific wavenumber regions that are responsible for the reduction in water as the sample is drying.

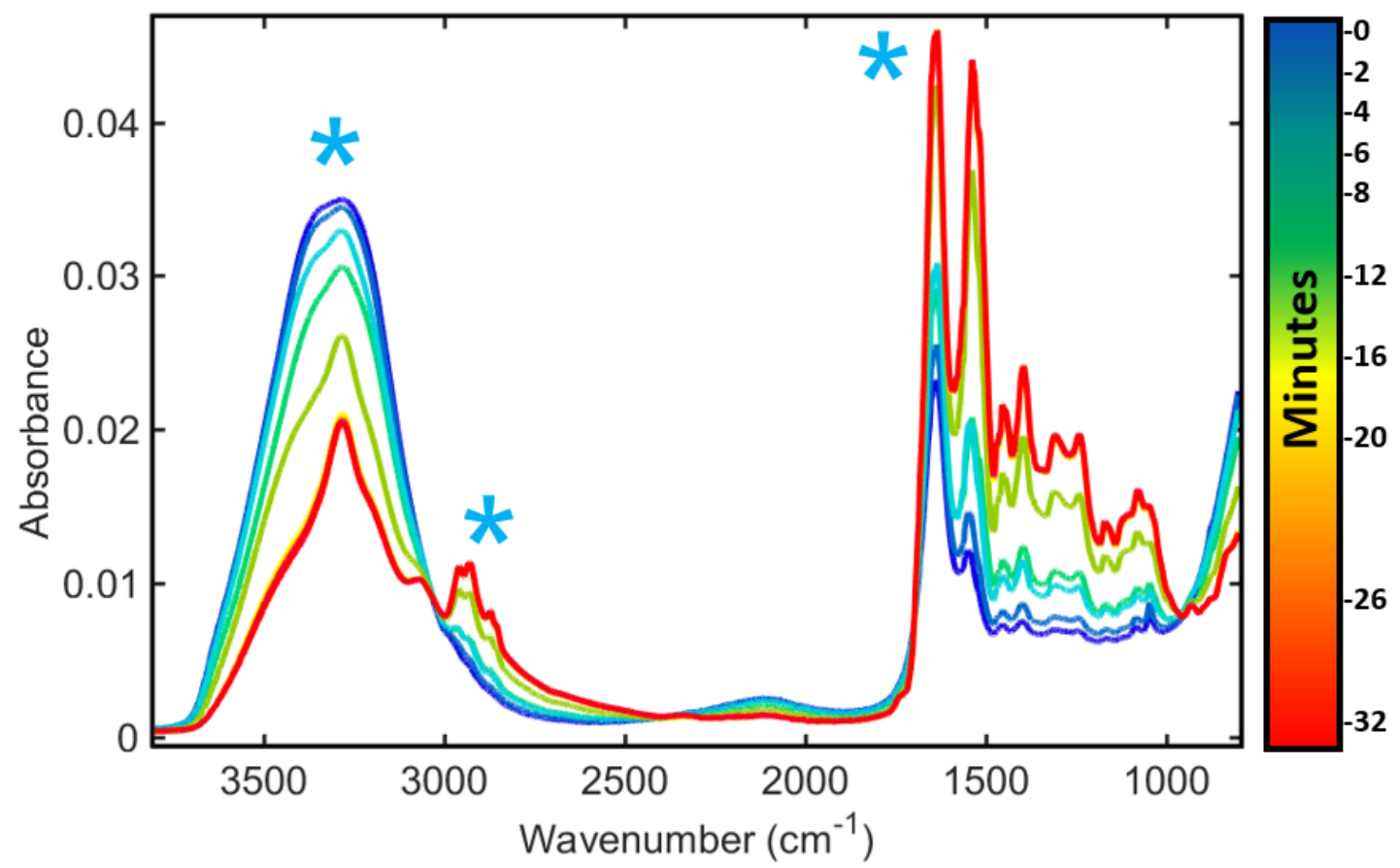

Figure 10: Infrared spectra of human pooled serum over an extended time period from 0-32 minutes on a diamond IRE. Blue asterisks highlight regions where distinct spectral changes are made between wet and dry samples.

\subsection{Accelerated drying on diamond and silicon}

It is to be expected that any type of forced drying protocol would accelerate the drying times of biofluid specimens quicker than if left to dry in ambient conditions. For diamond IREs this is evidenced by a quicker reduction in the $\mathrm{OH}$ bend region of all drying methods at $35^{\circ} \mathrm{C}$ with added fan assistance (Figure S9, SI). The average area under the curve (AUC) at the OH band was calculated for four-time intervals of each of the 12 drying regimes tested. Incorporating a fan and heater combination was deemed the most optimal. These results have been presented in Table 3 as the reduction in $\mathrm{OH}$ is a direct indicator of the drying process. 
Table 3: Area under the curve values at the $\mathrm{OH}$ band at different temperatures and time intervals.

\begin{tabular}{|c|c|c|c|c|c|}
\hline \multirow{3}{*}{ Temp $\left({ }^{\circ} \mathrm{C}\right)$} & \multirow{3}{*}{ Time $(\mathrm{min})$} & \multicolumn{4}{|c|}{ Air Flow $($ CFM) } \\
\cline { 2 - 6 } & & 0 & 9 & 60 & 70 \\
\hline \multirow{3}{*}{25} & 0 & 28.7 & 30.7 & 27.9 & 29.3 \\
\cline { 2 - 6 } & 2 & 28.3 & 29.2 & 16.4 & 18.5 \\
\cline { 2 - 6 } & 4 & 22.8 & 19.7 & 16.0 & 15.8 \\
\hline \multirow{3}{*}{30} & 6 & 19.8 & 15.2 & 15.9 & 15.7 \\
\cline { 2 - 6 } & 2 & 28.7 & 26.8 & 21.9 & 22.6 \\
\cline { 2 - 6 } & 4 & 16.9 & 14.0 & 13.0 & 12.9 \\
\hline \multirow{3}{*}{35} & 6 & 12.9 & 12.5 & 12.6 & 12.4 \\
\cline { 2 - 6 } & 2 & 27.8 & 12.3 & 12.7 & 12.4 \\
\cline { 2 - 6 } & 2 & 14.2 & 11.9 & 11.9 & 11.9 \\
\cline { 2 - 6 } & 4 & 12.3 & 11.2 & 11.8 & 11.8 \\
\cline { 2 - 6 } & 6 & 11.1 & 10.9 & 12.0 & 11.9 \\
\hline
\end{tabular}

Larger $\mathrm{OH}$ curve areas indicate a higher presence of water in the sample, whereas low $\mathrm{OH}$ AUC represents a more dried state. Temperature alone appears to have a much larger impact on drying rate than fan usage, although when combined the effectiveness of each technique is greatly amplified. Samples can be dried efficiently in 2 minutes with the more powerful airflow rate. While there appears to be significant improvements in drying time when increasing fan rates from $9 \mathrm{CFM}$ to $60 \mathrm{CFM}$, there does not seem to be substantial benefit in increasing the fan speed to $70 \mathrm{CFM}$. The greatest improvement in drying speed is still due to gentle heating, but the only way to ensure complete drying in two minutes is to use a combination of fans and heating. This is observed by the minor changes in area under the $\mathrm{OH}$ curve following the 2-minute mark for droplets subjected to both heating above $30^{\circ} \mathrm{C}$ and forced air drying above 60 CFM. Table 4 shows the relative standard deviation (RSD\%) of the area under the $\mathrm{OH}$ bend 
for each drying regime tested to help establish which method is most reproducible, or to reveal any methods that might inadvertently be introducing variance into the sample. A less discernible pattern has emerged making the distinction of the most repeatable method less clear cut. In general, wet samples have poorer reproducibility perhaps due to higher scattering, less control over sample biomolecular distribution and sample thickness, and dispersion effects [44]. RSD\% values for tests carried out at $25{ }^{\circ} \mathrm{C}$ and $9 \mathrm{CFM}$ appear unreliable, as they are both very low at time zero $(0.4 \%)$ and very high at four minutes $(17.7 \%)$. Other values on Table 4 indicate that the use of a fan generally improves sample reproducibility with some of the lowest RSD\% values obtained at 70 CFM across all heater settings. It is not clear whether heating alone improves reproducibility of the dried films, but heating in combination with fan-assisted drying does seem to improve RSD\% values. The droplet displacement caused by airflow from the fan may be responsible for improvements in reproducibility as this would result in more consistent sample coverage within the well, forcing samples into uniform thicknesses and disrupting the normal mechanisms by which the 'coffee-ring' phenomena takes place [34].

Table 4: $\mathrm{RSD}(\%)$ of the $\mathrm{OH}$ band at different temperature and time intervals.

\begin{tabular}{|c|c|c|c|c|c|}
\hline \multirow{3}{*}{ Temp $\left({ }^{\circ} \mathrm{C}\right)$} & \multirow{3}{*}{ Time $(\mathrm{min})$} & \multicolumn{4}{|c|}{ Air Flow $($ CFM) } \\
\cline { 2 - 6 } & & 0 & 9 & 60 & 70 \\
\hline \multirow{3}{*}{25} & 0 & 17.9 & 0.4 & 8.5 & 3.8 \\
\cline { 2 - 6 } & 2 & 12.3 & 3.2 & 8.8 & 23.6 \\
\cline { 2 - 6 } & 4 & 32.2 & 17.7 & 5.9 & 4.2 \\
\hline \multirow{3}{*}{30} & 6 & 32.6 & 3.3 & 5.4 & 4.4 \\
\cline { 2 - 6 } & 2 & 16.5 & 10.7 & 20.8 & 5.5 \\
\cline { 2 - 6 } & 4 & 32.2 & 21.8 & 5.6 & 7.3 \\
\cline { 2 - 6 } & 6 & 10.3 & 6.9 & 5.5 & 8.4 \\
\hline \multirow{3}{*}{35} & 0 & 6.9 & 21.0 & 10.6 & 11.9 \\
\cline { 2 - 6 } & 2 & 26.0 & 4.5 & 9.7 & 5.9 \\
\cline { 2 - 6 } & 4 & 11.2 & 5.4 & 9.7 & 5.8 \\
\hline
\end{tabular}




\begin{tabular}{|l|l|l|l|l|l|}
\hline & 6 & 4.8 & 6.6 & 9.3 & 6.0 \\
\hline
\end{tabular}

Sample preparation with conventional ATR-FTIR upon a fixed diamond IRE is a severely time limiting factor, thus batched drying is a highly promising solution. Therefore, similar drying methods were applied to SIREs in an attempt to optimise the batch drying protocol. An incubator was used to as an environment that could uniformly heat numerous SIREs simultaneously. The effectiveness of this new approach was tested by placing SIREs into the incubator $\left(30^{\circ} \mathrm{C}\right)$, under a fan outputting $60 \mathrm{CFM}$ airflow for $0,2,4,6,8,16$, and 32 minutes. As before, samples can be dried rapidly in at least two minutes. Table 5 highlights the area under the $\mathrm{OH}$ curve and the corresponding $\mathrm{RSD} \%$ at each time interval. The AUC decreases rapidly after two minutes and remains relatively stable after this period. RSD\% also improves dramatically after 2 minutes, and remains low up until 32 minutes have elapsed.

Table 5: AUC and RSD\% values for the $\mathrm{OH}$ band from the spectra obtained from silicon IREs after incubation at $30{ }^{\circ} \mathrm{C}$ and under a fan at a speed of $60 \mathrm{CFM}$.

\begin{tabular}{|c|c|c|c|c|c|c|c|}
\hline Time (min) & 0 & 2 & 4 & 6 & 8 & 16 & 32 \\
\hline AUC of OH stretch & 12.9 & 7.4 & 7.2 & 7.1 & 7.1 & 6.9 & 7.0 \\
\hline RSD (\%) & 39.3 & 2.7 & 1.8 & 3.7 & 1.8 & 3.6 & 1.3 \\
\hline
\end{tabular}

Most drying strategies described in this section can rapidly remove water content from serum samples deposited upon IRE sample sites with good reproducibility. In particular, moderate heating $\left(30^{\circ} \mathrm{C}\right.$ or $\left.35^{\circ} \mathrm{C}\right)$ is capable of reducing drying times to approximately 2 minutes, and fan assisted drying (60 CFM or $70 \mathrm{CFM}$ ) is effective in maintaining and perhaps improving spectral quality of dried serum films. Humidity is another important parameter that should be kept consistent across batches of drying serum samples. Bou Zeid describes the differences in morphology of whole blood films which have dried under various relative humidity conditions [45]. Fracture patterns are much more prevalent in films dried in lower humidity may increase 
scattering prevalence in FTIR analysis thereby increasing spectral noise. Furthermore, a study has shown that constant temperature humidity chambers set to $50 \%$ humidity and $75{ }^{\circ} \mathrm{C}$ can be used to homogeneously distribute droplet precipitates with rapid evaporation times, although optimal humidity conditions for serum would have to be independently investigated [46]. Numerous studies describe the impact drying patterns can have on the resultant spectra of samples studied using FTIR [34,36,47]. In particular, non-uniformity in either sample thickness or biomolecular distribution are primary causes of poor spectral quality. Sustained shear forces from air flow generated by the fans will help smooth surface irregularities and maintain a uniform sample thickness. Heating of droplets is also known to promote uniform precipitation of molecular constituents following evaporation [46]. The benefits both heat and air flow drying mechanisms have on spectral quality is evident in the findings presented here signifying that a combination of both fans and heaters can vastly reduce sample preparation time of serum for spectral analysis, but also reduce extraneous sample variance. Ultimately, this demonstrates the viability of batched sample preparation using SIREs for high-throughput ATR-FTIR. Batch processing optical sample slides should effectively minimise the variations observed through complex droplet dehydration, allowing a more controlled protocol for the drying dynamics of serum.

\section{Conclusions}

With a reported two-thirds of clinical laboratory issues occurring in the pre-analytical phase [18], it seems imperative that the biomedical spectroscopy field aims to standardise and optimise sample collection and preparation protocols, in order to facilitate successful translation into clinical practice. Here we have examined various pre-analytical factors that have the potential to become translational barriers. Our results suggest that many of these factors do not have a profound impact on spectral quality and/or reproducibility, such as altering the centrifugation parameters and usage of various blood collection tubes. 
Investigating three different blood collection tubes and various centrifugal times and speeds has minimal impact on the spectral results providing blood is collected and serum is successfully produced. Short-term storage at $4{ }^{\circ} \mathrm{C}$ (up to 7 days) prior to freezing serum samples does not have a significant detrimental effect to the integrity of the biomolecular components. Similar to previous findings after storing frozen samples for 9 months [37], we found longer term storage at $-80{ }^{\circ} \mathrm{C}$ for a period of 28 months also illustrated no significant effects despite previous studies describing the degradation of blood-based biomolecules due to storage conditions [29].

Intra- and inter-operator variability is a common theme in clinical scenarios [48-51], but with rigorous analyst training - and provided the standard operating procedures are followed for each analysis - these effects will be minimal. Biofluid volumes do not have a substantial effect on spectra, however it is recommended to use an appropriate amount depending on the IRE for analysis.

Controlling the environment of the biofluids whilst they are drying and immediately prior to analysis will enable better reproducibility by removing any temperature and humidity variations which impacts on the dynamic of the droplet $[34,45,52]$. If a traditional fixed diamond IRE is used, fan-assisted drying will enable higher reproducibility and throughput. Likewise, disposable IREs may benefit from incubation-based drying processes.

Successful implementation of the technique will depend on thorough experimental design and relevance to the particular clinical application. It is crucial that we ensure spectroscopic analysis is based on the detection the true molecular and pathological signatures rather than potentially detrimental external influences. Herein we can conclude that spectroscopic analysis is robust, reliable and will not be influenced by variations in pre-analytical experimental design, differences between operators within one laboratory or between different laboratories. 


\section{Acknowledgements}

The authors would like to thank the EPSRC (EP/L505080/1) for funding.

\section{References}

[1] M. Baker, Biophotonics: Vibrational Spectroscopic Diagnostics, IOP Publishing, 2016. https://doi.org/10.1088/978-1-6817-4071-3.

[2] A. Sala, D.J. Anderson, P.M. Brennan, H.J. Butler, J.M. Cameron, M.D. Jenkinson, C. Rinaldi, A.G. Theakstone, M.J. Baker, Biofluid Diagnostics by FTIR Spectroscopy: A Platform Technology for Cancer Detection, Cancer Lett. (2020) S0304383520300835. https://doi.org/10.1016/j.canlet.2020.02.020.

[3] M.J. Baker, S.R. Hussain, L. Lovergne, V. Untereiner, C. Hughes, R.A. Lukaszewski, G. Thiéfin, G.D. Sockalingum, Developing and understanding biofluid vibrational spectroscopy: a critical review, Chem. Soc. Rev. 45 (2016) 1803-1818.

[4] B.R. Smith, K.M. Ashton, A. Brodbelt, T. Dawson, M.D. Jenkinson, N.T. Hunt, D.S. Palmer, M.J. Baker, Combining random forest and 2D correlation analysis to identify serum spectral signatures for neuro-oncology, Analyst. 141 (2016) 3668-3678.

[5] K. Beaumont, F. Betsou, Immunological Fingerprinting Method for Differentiation of Serum Samples in Research-Oriented Biobanks, Clin. Vaccine Immunol. 17 (2010) 735-740. https://doi.org/10.1128/CVI.00499-09.

[6] J. Backhaus, R. Mueller, N. Formanski, N. Szlama, H.-G. Meerpohl, M. Eidt, P. Bugert, Diagnosis of breast cancer with infrared spectroscopy from serum samples, Vib. Spectrosc. 52 (2010) 173-177. https://doi.org/10.1016/j.vibspec.2010.01.013.

[7] K. Gajjar, J. Trevisan, G. Owens, P.J. Keating, N.J. Wood, H.F. Stringfellow, P.L. Martin-Hirsch, F.L. Martin, Fourier-transform infrared spectroscopy coupled with a classification machine for the analysis of blood plasma or serum: a novel diagnostic approach for ovarian cancer, The Analyst. 138 (2013) 3917. https://doi.org/10.1039/c3an36654e.

[8] J. Ollesch, M. Heinze, H.M. Heise, T. Behrens, T. Brüning, K. Gerwert, It's in your blood: spectral biomarker candidates for urinary bladder cancer from automated FTIR spectroscopy: Spectral cancer biomarkers from high-throughput FTIR spectroscopy, J. Biophotonics. 7 (2014) 210-221. https://doi.org/10.1002/jbio.201300163.

[9] I. Maitra, C.L.M. Morais, K.M.G. Lima, K.M. Ashton, R.S. Date, F.L. Martin, Attenuated total reflection Fourier-transform infrared spectral discrimination in human bodily fluids of oesophageal transformation to adenocarcinoma, The Analyst. (2019) 10.1039.C9AN01749F. https://doi.org/10.1039/C9AN01749F.

[10] J.R. Hands, K.M. Dorling, P. Abel, K.M. Ashton, A. Brodbelt, C. Davis, T. Dawson, M.D. Jenkinson, R.W. Lea, C. Walker, M.J. Baker, Attenuated Total Reflection Fourier Transform Infrared (ATR-FTIR) spectral discrimination of brain tumour severity from serum samples: Serum spectroscopy gliomas, J. Biophotonics. 7 (2014) 189-199. https://doi.org/10.1002/jbio.201300149.

[11] J.R. Hands, G. Clemens, R. Stables, K. Ashton, A. Brodbelt, C. Davis, T.P. Dawson, M.D. Jenkinson, R.W. Lea, C. Walker, M.J. Baker, Brain tumour differentiation: rapid stratified serum diagnostics via attenuated total reflection Fourier-transform infrared 
spectroscopy, J. Neurooncol. 127 (2016) 463-472. https://doi.org/10.1007/s11060-0162060-x.

[12] H.J. Butler, P.M. Brennan, J.M. Cameron, D. Finlayson, M.G. Hegarty, M.D. Jenkinson, D.S. Palmer, B.R. Smith, M.J. Baker, Development of high-throughput ATR-FTIR technology for rapid triage of brain cancer, Nat. Commun. 10 (2019) 4501. https://doi.org/10.1038/s41467-019-12527-5.

[13] J.M. Cameron, H.J. Butler, B.R. Smith, M.G. Hegarty, M.D. Jenkinson, K. Syed, P.M. Brennan, K. Ashton, T. Dawson, D.S. Palmer, M.J. Baker, Developing infrared spectroscopic detection for stratifying brain tumour patients: glioblastoma multiforme vs. lymphoma, The Analyst. 144 (2019) 6736-6750.

https://doi.org/10.1039/C9AN01731C.

[14] M. Paraskevaidi, C.L.M. Morais, K.M.G. Lima, J.S. Snowden, J.A. Saxon, A.M.T. Richardson, M. Jones, D.M.A. Mann, D. Allsop, P.L. Martin-Hirsch, F.L. Martin, Differential diagnosis of Alzheimer's disease using spectrochemical analysis of blood, Proc. Natl. Acad. Sci. 114 (2017) E7929-E7938. https://doi.org/10.1073/pnas.1701517114.

[15] L. Lovergne, P. Bouzy, V. Untereiner, R. Garnotel, M.J. Baker, G. Thiéfin, G.D. Sockalingum, Biofluid infrared spectro-diagnostics: pre-analytical considerations for clinical applications, Faraday Discuss. 187 (2016) 521-537. https://doi.org/10.1039/C5FD00184F.

[16] M.J. Baker, H.J. Byrne, J. Chalmers, P. Gardner, R. Goodacre, A. Henderson, S.G. Kazarian, F.L. Martin, J. Moger, N. Stone, J. Sulé-Suso, Clinical applications of infrared and Raman spectroscopy: state of play and future challenges, The Analyst. 143 (2018) 1735-1757. https://doi.org/10.1039/C7AN01871A.

[17] H.J. Byrne, M. Baranska, G.J. Puppels, N. Stone, B. Wood, K.M. Gough, P. Lasch, P. Heraud, J. Sulé-Suso, G.D. Sockalingum, Spectropathology for the next generation: Quo vadis?, The Analyst. 140 (2015) 2066-2073. https://doi.org/10.1039/C4AN02036G.

[18] P. Carraro, M. Plebani, Errors in a Stat Laboratory: Types and Frequencies 10 Years Later, Clin. Chem. 53 (2007) 1338-1342. https://doi.org/10.1373/clinchem.2007.088344.

[19] O.Y. Buowari, Complications of venepuncture, Adv. Biosci. Biotechnol. 04 (2013) 126-128. https://doi.org/10.4236/abb.2013.41A018.

[20] N. Dhingra, Safe Injection Global Network, World Health Organization, WHO guidelines on drawing blood: best practices in phlebotomy, 2010. http://www.ncbi.nlm.nih.gov/books/NBK138650/ (accessed December 2, 2019).

[21] J. Cadamuro, C. Mrazek, A.B. Leichtle, U. Kipman, T.K. Felder, H. Wiedemann, H. Oberkofler, G.M. Fiedler, E. Haschke-Becher, Influence of centrifugation conditions on the results of 77 routine clinical chemistry analytes using standard vacuum blood collection tubes and the new BD-Barricor tubes, Biochem. Medica. 28 (2018) 010704. https://doi.org/10.11613/BM.2018.010704.

[22] J.H. Livesey, M.J. Ellis, M.J. Evans, Pre-analytical requirements, Clin. Biochem. Rev. 29 Suppl 1 (2008) S11-15.

[23] L. Wiegmann, D.A. de Zélicourt, O. Speer, A. Muller, J.S. Goede, B. Seifert, V. Kurtcuoglu, Influence of Standard Laboratory Procedures on Measures of Erythrocyte Damage, Front. Physiol. 8 (2017) 731. https://doi.org/10.3389/fphys.2017.00731.

[24] G. Lippi, G. Luca Salvagno, M. Montagnana, G. Brocco, G. Cesare Guidi, Influence of hemolysis on routine clinical chemistry testing, Clin. Chem. Lab. Med. CCLM. 44 (2011). https://doi.org/10.1515/CCLM.2006.054. 
[25] L.-H. Huang, P.-H. Lin, K.-W. Tsai, L.-J. Wang, Y.-H. Huang, H.-C. Kuo, S.-C. Li, The effects of storage temperature and duration of blood samples on DNA and RNA qualities, PLOS ONE. 12 (2017) e 0184692.

https://doi.org/10.1371/journal.pone.0184692.

[26] A. Hubel, R. Spindler, A.P.N. Skubitz, Storage of Human Biospecimens: Selection of the Optimal Storage Temperature, Biopreservation Biobanking. 12 (2014) 165-175. https://doi.org/10.1089/bio.2013.0084.

[27] J.B. Vaught, M.K. Henderson, Biological sample collection, processing, storage and information management, IARC Sci. Publ. (2011) 23-42.

[28] S.S. Tworoger, Collection, Processing, and Storage of Biological Samples in Epidemiologic Studies: Sex Hormones, Carotenoids, Inflammatory Markers, and Proteomics as Examples, Cancer Epidemiol. Biomarkers Prev. 15 (2006) 1578-1581. https://doi.org/10.1158/1055-9965.EPI-06-0629.

[29] S. Enroth, G. Hallmans, K. Grankvist, U. Gyllensten, Effects of Long-Term Storage Time and Original Sampling Month on Biobank Plasma Protein Concentrations, EBioMedicine. 12 (2016) 309-314. https://doi.org/10.1016/j.ebiom.2016.08.038.

[30] J.-E. Lee, S.Y. Kim, S.-Y. Shin, Effect of Repeated Freezing and Thawing on Biomarker Stability in Plasma and Serum Samples, Osong Public Health Res. Perspect. 6 (2015) 357-362. https://doi.org/10.1016/j.phrp.2015.11.005.

[31] F. Bonnier, F. Petitjean, M.J. Baker, H.J. Byrne, Improved protocols for vibrational spectroscopic analysis of body fluids: Improved protocols for vibrational spectroscopic analysis of body fluids, J. Biophotonics. 7 (2014) 167-179. https://doi.org/10.1002/jbio.201300130.

[32] R.D. Deegan, Pattern formation in drying drops, Phys. Rev. E. 61 (2000) 475.

[33] R.D. Deegan, O. Bakajin, T.F. Dupont, G. Huber, S.R. Nagel, T.A. Witten, Capillary Flow as the Cause of Ring Stains from Dried Liquid Drops, Nature. 389 (1997) 827829.

[34] J.M. Cameron, H.J. Butler, D.S. Palmer, M.J. Baker, Biofluid spectroscopic disease diagnostics: A review on the processes and spectral impact of drying, J. Biophotonics. 11 (2018) e201700299. https://doi.org/10.1002/jbio.201700299.

[35] C. Hughes, M. Brown, G. Clemens, A. Henderson, G. Monjardez, N.W. Clarke, P. Gardner, Assessing the challenges of FTIR spectroscopic analysis of blood serum, J. Biophotonics. 7 (2014) 180-188. https://doi.org/10.1002/jbio.201300167.

[36] L. Lovergne, G. Clemens, V. Untereiner, R.A. Lukaszweski, G.D. Sockalingum, M.J. Baker, Investigating optimum sample preparation for infrared spectroscopic serum diagnostics, Anal Methods. 7 (2015) 7140-7149. https://doi.org/10.1039/C5AY00502G.

[37] L. Lovergne, J. Lovergne, P. Bouzy, V. Untereiner, M. Offroy, R. Garnotel, G. Thiéfin, M.J. Baker, G.D. Sockalingum, Investigating pre-analytical requirements for serum and plasma based infrared spectro-diagnostic, J. Biophotonics. 12 (2019). https://doi.org/10.1002/jbio.201900177.

[38] Z. Movasaghi, S. Rehman, Dr.I. ur Rehman, Fourier Transform Infrared (FTIR) Spectroscopy of Biological Tissues, Appl. Spectrosc. Rev. 43 (2008) 134-179. https://doi.org/10.1080/05704920701829043.

[39] D. Alberghina, S. Casella, C. Giannetto, S. Marafioti, G. Piccione, Effect of storage time and temperature on the total protein concentration and electrophoretic fractions in equine serum, Can. J. Vet. Res. Rev. Can. Rech. Veterinaire. 77 (2013) 293-296.

[40] M.J. Baker, J. Trevisan, P. Bassan, R. Bhargava, H.J. Butler, K.M. Dorling, P.R. Fielden, S.W. Fogarty, N.J. Fullwood, K.A. Heys, C. Hughes, P. Lasch, P.L. MartinHirsch, B. Obinaju, G.D. Sockalingum, J. Sulé-Suso, R.J. Strong, M.J. Walsh, B.R. Wood, P. Gardner, F.L. Martin, Using Fourier transform IR spectroscopy to analyze 
biological materials, Nat. Protoc. 9 (2014) 1771-1791.

https://doi.org/10.1038/nprot.2014.110.

[41] M.G. Bridelli, Fourier Transform Infrared Spectroscopy in the Study of Hydrated Biological Macromolecules, in: G.S. Nikolic, M.D. Cakic, D.J. Cvetkovic (Eds.), Fourier Transforms - High-Tech Appl. Curr. Trends, InTech, 2017. https://doi.org/10.5772/66576.

[42] K.L.A. Chan, An Introduction to Infra-red and Raman Spectroscopies for Pharmaceutical and Biomedical Studies, in: Biomol. Bioanal. Tech., John Wiley \& Sons, Ltd, Chichester, UK, 2019: pp. 307-331. https://doi.org/10.1002/9781119483977.ch13.

[43] M. Hancer, R.P. Sperline, J.D. Miller, Anomalous Dispersion Effects in the IR-ATR Spectroscopy of Water, Appl. Spectrosc. 54 (2000) 138-143. https://doi.org/10.1366/0003702001948222.

[44] J. Ollesch, S.L. Drees, H.M. Heise, T. Behrens, T. Brüning, K. Gerwert, FTIR spectroscopy of biofluids revisited: an automated approach to spectral biomarker identification, The Analyst. 138 (2013) 4092. https://doi.org/10.1039/c3an00337j.

[45] W. Bou Zeid, D. Brutin, Influence of relative humidity on spreading, pattern formation and adhesion of a drying drop of whole blood, Colloids Surf. Physicochem. Eng. Asp. 430 (2013) 1-7. https://doi.org/10.1016/j.colsurfa.2013.03.019.

[46] Y. Li, Q. Yang, M. Li, Y. Song, Rate-dependent interface capture beyond the coffeering effect, Sci. Rep. 6 (2016). https://doi.org/10.1038/srep24628.

[47] F. Bonnier, H. Blasco, C. Wasselet, G. Brachet, R. Respaud, L.F.C.S. Carvalho, D. Bertrand, M.J. Baker, H.J. Byrne, I. Chourpa, Ultra-filtration of human serum for improved quantitative analysis of low molecular weight biomarkers using ATR-IR spectroscopy, The Analyst. 142 (2017) 1285-1298. https://doi.org/10.1039/C6AN01888B.

[48] Z.B. Popović, J.D. Thomas, Assessing observer variability: a user's guide, Cardiovasc. Diagn. Ther. 7 (2017) 317-324. https://doi.org/10.21037/cdt.2017.03.12.

[49] S. Shahangian, R.D. Cohn, Variability of Laboratory Test Results, Am. J. Clin. Pathol. 113 (2000) 521-527. https://doi.org/10.1309/LF4J-0B04-HUYK-NKE2.

[50] R. Phetsouvanh, T. Thojaikong, P. Phoumin, B. Sibounheuang, K. Phommasone, V. Chansamouth, S.J. Lee, P.N. Newton, S.D. Blacksell, Inter- and Intra-Operator Variability in the Reading of Indirect Immunofluorescence Assays for the Serological Diagnosis of Scrub Typhus and Murine Typhus, Am. J. Trop. Med. Hyg. 88 (2013) 932-936. https://doi.org/10.4269/ajtmh.12-0325.

[51] M.R. Elashoff, R. Nuttall, P. Beineke, M.H. Doctolero, M. Dickson, A.M. Johnson, S.E. Daniels, S. Rosenberg, J.A. Wingrove, Identification of Factors Contributing to Variability in a Blood-Based Gene Expression Test, PLoS ONE. 7 (2012) e40068. https://doi.org/10.1371/journal.pone.0040068.

[52] W. Bou Zeid, J. Vicente, D. Brutin, Influence of evaporation rate on cracks' formation of a drying drop of whole blood, Colloids Surf. Physicochem. Eng. Asp. 432 (2013) 139-146. https://doi.org/10.1016/j.colsurfa.2013.04.044. 